\title{
Study the Effect of the Risk Factors in the Estimation of the Breast Cancer Risk Score Using Machine Learning
}

\author{
Sam Khozama ${ }^{1 *}$, Ali Mahmoud Mayya ${ }^{2}$
}

\begin{abstract}
Objective: Early prediction of breast cancer is one of the most essential fields of medicine. Many studies have introduced prediction approaches to facilitate the early prediction and estimate the future occurrence based on mammography periodic tests. In the current research, we introduce a novel machine learning tool for the early prediction of breast cancer. Methods: Three basic resources are used to identify the most essential risk factors; including the BCSC (Breast Cancer Surveillance Consortium) dataset, a medical questionnaire, and multiple international breast cancer reports. The BCSC dataset has been normalized and balanced; consequently, the questionnaire and the medical reports are analyzed in order to define the degree of importance and a potential weight factor of each risk factor. These weights are used to scale risk factors and then the optimizable tree-based ML model is trained using the balanced weighted risk factors datasets. Results: Three balanced versions of the BCSC dataset are used; oversampled, down-sampled and mixed datasets. Each risk factor has a weight (1,2 or 4) assigned based on a mathematical modelling of the questionnaire and the international breast cancer reports. The experiments are applied on the weighted and non-weighted versions of the database, and they indicate that the performance increases significantly by using the weighted version of the risk factors. The tests prove that the down-weighting of the non-essential risk factor increases the accuracy and reduces errors. The overall accuracy of the weighted balanced datasets reaches $100 \%, 95.8 \%$ and $95.9 \%$ for down-sampled, oversampled and mixed datasets respectively. Conclusion: Weighting the risk factors of the BCSC dataset improves the performance by increasing the accuracy and reducing the false rejection and false discovery rates for all versions of balanced datasets. The weighting approach can also be used to improve the estimation score of breast cancer by scaling the individual scores of risk factors.
\end{abstract}

Keywords: Breast cancer- cancer prediction- machine learning- risk factors

Asian Pac J Cancer Prev, 22 (11), 3543-3551

\section{Introduction}

Nowadays, data analysis is one of the most developing fields of computer science due to the fact that the size of datasets is exponentially increasing day after day. Cancer prediction is one of those fields, using data analysis and Machine Learning (ML) algorithms for the estimation of cancer (Faith, 2020) (Patil, 2020; Kamal, 2020). ML techniques can improve the performance of cancer prediction, the estimation accuracy of which has increased significantly (15\%-20\%) due to using the ML algorithm during the last years (Kourou et al., 2015). Breast cancer prediction itself can be used to define those potentially high-risk women and guide them to improve their lifestyle, avoiding future therapy and costs (Colditz and Wei, 2015).

Cancer prediction is generally based on risk factors (Ahmad and Mayya, 2020). Half of the cancer cases are caused by some known risk factors (Laky, 2020). For breast cancer, many risk factors, such as early menarche, late menopause, obesity, age at first birth, and hormone therapy affect the exposure period of breast tissue to hormones that lead to cancer (American Cancer Society, 2019).

In the field of breast cancer predictions, some studies used the logistic regression approaches (Bernal et al., 2017; Oyewola et al., 2017; Westerdijk, 2018; Teja et al., 2020), while other studies used neural networks (Wang and Yoon, 2015; Kourou et al., 2015; Hou et al. 2020). Other data mining algorithms were used like decision trees (Rajendran et al., 2020), Naïve Bayes methods (Rajendran et al., 2020; Shieh et al., 2016; Williams et al., 2016), Support Vector Machines (Westerdijk, 2018; Mochen and Sundararajan, 2018; Vard et al., 2018), Random Forests (RF) (Oyewola et al., 2017; Westerdijk, 2018; Hou et al., 2020; Rajendran et al., 2020), optimization algorithms (Vard et al., 2018), etc.

For breast cancer estimation research, many datasets, like the Breast Cancer Surveillance Consortium dataset (BCSC dataset, 2021), consisting of 280,660 records, had been used in many pieces of research (Rajendran et al.,

${ }^{1}$ Department of Information Technology and Bionics, Pázmány Péter Catholic University, Budapest, Hungary. ${ }^{2}$ Department of Computer Engineering, Tishreen University, Lattakia, Syria.*For Correspondence: khozama.sam@itk.ppke.hu 
2020; Shieh et al., 2016; BCSC dataset, 2021; Williams et al., 2016). Another international dataset is the Breast Cancer Information Management System (BCIMS) dataset, consisting of 16,000, cases (Peng et al., 2016) and was used by many studies such as Hou et al. (2020) and Zhong et al. (2020). Some other researchers collected their datasets from specialized medical centers or hospitals (Ming et al., 2020; Barlow et al., 2006).

Shieh et al., (2016) proposed a breast cancer prediction model using the information of the clinical and polygenic risks. The Bayes estimation and conditional logistic regression models are used together to study the common effect of ordinary and polygenic risk factors on the future risk of breast cancer. The researchers used 486 cases of the $\mathrm{BCSC}$ dataset and found that the prediction accuracy increased from $\mathrm{AUC}=0.62$ to $\mathrm{AUC}=0.65$ after adding the polygenic risk to the model. They concluded that $18 \%$ of the cases were classified as high-risk cases in the common model, while it was only $7 \%$ for the ordinary risk factors model.

Li and Sundararajan (2018) applied several ML approaches for the prediction of breast cancer. They used only 10,000 cases and eight risk factors of the BCSC dataset. SVM and Bayes classifiers were used for the final risk estimation and got accuracy results of $96.6 \%$ and $91.26 \%$ for SVM and Bayes classification, respectively.

In 2020, Rajendran et al., (2020) used the supervised ML algorithms on imbalanced class data for the prediction of breast cancer on the BCSC dataset. In order to perform balancing, they used three approaches: Synthetic Minority Oversampling, under-sampling and fusion of both techniques. They also used Bayes classifier, Bayes networks, Random Forests (RF), and random trees as classifiers. The best accuracy they obtained was $99.1 \%$, under False Positive (FP) equals 21\%. The problem with this research was that they used only 10,252 instances after applying the balancing techniques; besides that, the results showed low sensitivity of $78.1 \%$.

A new model for predicting breast cancer in Chinese women had been introduced by Hou et al., (2020). They used 7,127 cases of the Breast Cancer Information Management System (BCIMS) dataset and chose specific risk factors based on the fact that they must be known and collected by the same measurement techniques. Consequently, 10 risk factors had been chosen and different prediction models were used, like RF, deep neural networks DNN and XGBoost. They got an accuracy of 72.8 for both DNN and RF, while the XGBoost accuracy was $74.2 \%$.

The Evaluation of many ML classifiers for the prediction of breast cancer under incomplete datasets was suggested by Teja et al., (2020). They evaluated the RF, Logistic Regression (LR) and custom Neural Network (NN). The Area Under Curve (AUC) was used for the performance evaluation on the BCSC dataset. AUC achieved $0.645,0.634$ and 0.649 for LR, RF and $\mathrm{NN}$ respectively.

Ming et al., (2020) collected a breast cancer prediction dataset from Geneva University Hospitals. Their dataset included 112587 individuals and 14 variables. They applied different ML algorithms (like the Markov mixed model, adaptive boosting and RF) and obtained accuracy between $84.3 \%$ and $88.9 \%$. However, the dataset variables related not only to breast cancer but also to other tissues, so that more risk factors needed to be included.

Most previous studies did not consider the nature of the used breast cancer dataset. Each dataset has some properties that must be understood in order to get a proper accurate estimation as mentioned by the BCSC dataset (2021). For example, the BCSC dataset needs to consider the "count" as a very important variable in order to achieve correct results. Besides that, the BCSC dataset is unbalanced, so it needs a balancing step before any estimation model. The research aim is to develop a new tool for predicting breast cancer based on BCSC risk factors. We have taken into account the "count" variable for good estimation. In addition to that, balancing has been applied as a pre-processing step. The last new option that have been done is the weighting mechanism, in which a weight number of each risk factor is assigned in order to enhance the performance. The following paragraphs will contain the used materials and the proposed approach in detail. Finally, the results and discussion section will be introduced.

\section{Materials and Methods}

\section{Dataset}

In the current research, the BCSC dataset is used. It includes 2809660 records and 12 risk factors, which are described in Table 1. Besides these risk factors, the dataset includes a variable called "count", which holds the frequency of each record within the dataset, as mentioned in the BCSC dataset (2021).

\section{Proposed system}

The proposed risk-estimation model of breast cancer is described in Figure 1 so that the BCSC dataset is obtained from http://www.bcsc-research.org/, and all risk factors are used. First, the dataset is normalized to ensure that all risk factors initially have the same effect on the final risk estimation. The normalization is done using Equation 1:

$$
\begin{aligned}
& \text { Risk_factor }_{i}=\text { Risk_factor }_{i} / \max \left(\text { Risk_factor }_{i}\right) \\
& \mathrm{i}=1,2, \ldots, \mathrm{M}
\end{aligned}
$$

Where $\mathrm{M}$ is the number of risk factors. The normalization step makes the value of each risk factor ranging from 0 to 1 .

The second step is balancing, in which the dataset must be manipulated in order to achieve the balancing between target categories. The original BCSC dataset has two target categories ( 0 : no cancer, 1 : cancer). While the " 1 " category has only $3.32 \%$, the " 0 " category has $96.68 \%$ of all samples, which is why the BCSC is extremely unbalanced. The minimal appearance of the minor category in the unbalanced datasets leads any classifier to generate inaccurate predictions due to the inappropriate training (Somasundaram and Reddy, 2016). So for the original BCSC dataset, any classifier can produce more than $96 \%$ accuracy only if it has recognized 
all " 0 " class samples, even if all " 1 " class samples have been incorrectly estimated as " 0 ". To solve this problem, the so-called balancing mechanism is applied. Three different balancing approaches were applied. The first is oversampling, in which the samples of the minor class are duplicated many times so that their percentage increases. Duplication will enhance the training significantly. The second approach is down-sampling, in which some of the majority-class samples are removed until decreasing its percentage to the required value. As for the last approach, duplicating the minor class samples and eliminating some of the majority class samples are performed until achieving the desired balance.

To perform the third step, the weighting algorithm is applied. To achieve a good, accurate weighting, two branches were taken. First, a questionnaire of the risk factors listed in the BCSC dataset was created. The aim of this questionnaire was to establish medical knowledge, so it was sent to 40 specialist physicians working in the field of cancer treatment and diagnosis. Afterward, the results of the questionnaire were analyzed to define the expert's opinion of the impact of each risk factor in the final score of cancer risk. The second branch of this study investigated the international medical reports from which the recent discussions of breast cancer risk factors were obtained to define the impact from another point of view.

\section{Machine Learning Model selection}

After getting the final impact (weight) of each risk factor, the final step is the selection of the ML model. Multiple ML prediction algorithms are available, but the optimization tree model is chosen due to its ability to tune the hyperparameters, deal with missing or noisy data, and handle redundant attributes values (Apté and Weiss, 1997; Mantovani et al., 2018). The decision tree algorithm first considers all samples of the dataset as the root node. The basic challenges are selecting the best attribute to be the root node and then deciding to split the node into all attributes and select the one with the best split performance. Decision trees actually compute the Information Gain (IG) as illustrated in Equation 2 (Kelleher, 2020) across all possible attributes and then choose the attribute with the lowest IG. This means that the selected attribute is the one that separates the training samples the best.

$$
I G(T, a)=H(T)-H(T \mid a)=-\sum_{i=1}^{k} p_{i} \log _{2}\left(p_{i}\right)-\sum_{i=1}^{k}-p_{r}(i \mid a) \log _{2}\left(p_{r}(i \mid a)\right)
$$

Where $\mathrm{H}(\mathrm{T})$ is the entropy of the parent node of the tree $\mathrm{T}, \mathrm{H}(\mathrm{T} \mid \mathrm{a})$ is the entropy of the child node a (attribute a), $\mathrm{k}$ is the number of subsets generated by each split, pi is the percentage (probability) of class $i$ in the node $\mathrm{T}, \operatorname{pr}(\mathrm{i} \mid \mathrm{a})$ is the percentage of class $\mathrm{i}$ given that the split child (attribute) is a.

Now, for the optimizable tree classifier, three different parameters are tuned. These parameters are the criterion (the attribute selection measure), the splitter (the split strategy) and the maximum depth of a tree.

\section{Prediction Tool Design}

After getting the final optimizable decision tree classifier, the tool is built based on the trained model. The tool is designed using MATLAB App designer. Figure 2 shows the designed tool. After entering the values of all factors except "count", the tool searches into the BCSC dataset to find the match between the entered risk values and all records of the dataset. If a match is found, the corresponding count is considered as the count of the test sample. Otherwise, the count will be 1 .

\section{Results}

\section{The preprocessing of the risk estimation dataset}

Preprocessing of the risk estimation dataset includes two basic steps, which are normalization and balancing. Table 2 includes the results of the suggested balancing methods, where the majority class label is 0 (no cancer), and the minor class label is 1 (cancer risk).

For the oversampling approach, the " 1 " minor class has been duplicated five times until its percentage became $14.64 \%$, while the majority-class percentage became $85.36 \%$. For the down-sampling approach, we minimized the majority-class samples by a factor (3.524 times) until getting a $10.78 \%$ percentage for the minor class and $89.22 \%$ for the majority one. For the last approach, we duplicated the minor class samples and eliminated some of the majority class samples until getting $17.1 \%$ and $82.9 \%$ for the minor and majority classes, respectively.

\section{Risk factor weighting results}

In order to obtain the risk factors weights, the results of the questionnaire are analyzed and the questionnairebased degree of importance $\left(\mathrm{DOI}_{\mathrm{i}}^{\mathrm{q}}\right)$ of each risk factor is defined based on Equation 3:

$$
D O I_{i}^{q}=H_{i} * 0.6+M_{i} * 0.4
$$

Where $\mathrm{H}_{\mathrm{i}}$ and $\mathrm{M}_{\mathrm{i}}$ are the high-risk and medium-risk percentages of the risk factor i shown in Table 3.

Based on the analysis of the questionnaire, the following results are inferred:

The risk factors with the largest high-risk levels are number of first degree relatives with breast cancer (nrelbc), hormone therapy.

Age, menopause, density and race are the risk factors with the largest medium-risk levels.

Hispanic, breast procedure (brstproc), and surgical menopause have the lowest risk levels.

Factors with a high DOI (more than 0.4) are nrelbc, age, hormone therapy.

Some other risk factors like age at first birth, menopause, density, Body Mass Index (BMI), last mammogram before the index mammogram (lastmamm) and race have medium DOI (between 0.3 and 0.4 ) are

Other risk factors with a low DOI (less than 0.3) are Hispanic, brstproc and surgical menopause.

The international medical reports, on the other hand, indicate other opinions. So, we concluded the information about risk factors, and then this information was compiled and classified according to the number of times the factors were mentioned in the list of the essential risk factors (Ess_Numi), then within the list of the secondary-risk 
Table1. Dataset Risk factors Description

\begin{tabular}{|c|c|c|}
\hline No. & Risk Factor & Description \\
\hline 1 & Menopause & Pre $=0(23.47 \%)$, Post or age $>55=1(68.65 \%)$, Unknown $=9(7.6 \%)$ \\
\hline 2 & Age group & $\begin{array}{l}\text { Group1=35-39(1.79\%); Group2=40-44(12.1\%); Group3=45-49(16.18\%); Group4=50-54(17.9\%); } \\
\text { Group5=55-59(13.96); Group6 }=60-64(11.1 \%) ; \text { Group7 }=65-69(9.69 \%) ; \text { Group8=70-74(8.49\%); } \\
\text { Group9=75-79(6.06\%); Group10=80-84(2.91\%). }\end{array}$ \\
\hline 3 & Density & $\begin{array}{l}\text { Breast density: Almost entirely fatty: } 1(6.19 \%) \text {, Scattered fibro-glandular densities:2(32.69\%), } \\
\text { Heterogeneously dense:3(28.17\%), Extremely dense:4(5.68\%), 9:Unknown or other indexes(27.26\%) }\end{array}$ \\
\hline 4 & Race & $\begin{array}{l}1=\text { white }(72.63 \%) ; 2=\text { Asian } / \text { Pacific Islander }(4.3 \%) ; 3=\text { black }(5.08 \%) ; 4=\text { Native American } \\
(1.19 \%) ; 5=\text { other } / \operatorname{mixed}(0.9 \%) ; 9=\text { unknown }(15.87 \%)\end{array}$ \\
\hline 5 & Hispanic & No:0(73.1\%) Yes:1(6.58\%), Unknown:9(20.3\%) \\
\hline 6 & BMI & $\begin{array}{l}1=10-24.99(21.27 \%) ; 2=25-29.99(13.6 \%) ; 3=30-34.99(6.05 \%) ; 4=35 \text { or more }(3.25 \%) ; \\
9=\text { unknown(55.83\%) }\end{array}$ \\
\hline 7 & Age at first birth (agefirst) & $0=$ Age $<30(30.18 \%) ; 1=$ Age 30 or greater $(5.9 \%) ; 2=$ Nulliparous $(8.41 \%) ; 9=$ unknown $(55.51 \%)$ \\
\hline 8 & $\begin{array}{l}\text { Number of first degree } \\
\text { relatives with breast cancer } \\
\text { (nrelbc) }\end{array}$ & $0=$ zero $(71.81 \%) ; 1=$ one $(12.36 \%) ; 2=2$ or more $(0.65 \%) ; 9=$ unknown $(15.18 \%)$ \\
\hline 9 & $\begin{array}{l}\text { Previous breast procedure } \\
\text { (brstproc) }\end{array}$ & $0=\operatorname{no}(71.97 \%) ; 1=y e s(17.57 \%) ; 9=$ unknown $(10.46 \%)$ \\
\hline 10 & $\begin{array}{l}\text { last mammogram before the } \\
\text { index mammogram } \\
\text { (lastmamm) }\end{array}$ & $0=$ negative $(75.22 \%) ; 1=$ false positive $(1.42 \%) ; 9=$ unknown $(23.36 \%)$ \\
\hline 11 & Surgical menopause & $0=$ natural $(30 \%) ; 1=\operatorname{surgical}(17.86 \%) ; 9=$ unknown or not menopausal $(52.14 \%)$ \\
\hline 12 & Hormone therapy & $0=\operatorname{no}(30.47 \%) ; 1=$ yes $(28.56 \%) ; 9=$ unknown $(40.97 \%)$ \\
\hline 13 & Count & Frequency of each record in the dataset \\
\hline
\end{tabular}

factors (Sec_Numi), and the risk degree $\mathrm{DOI}^{\mathrm{R}}{ }_{\mathrm{i}}$ was calculated according to Equation 4:

$$
\operatorname{DOI}_{i}^{R}=\frac{1}{4} *\left(0.9 * \sum_{\substack{j=1 \\ 1 \leq j \leq n}}^{n} \operatorname{ESS}_{\mathrm{Num}_{i}}+0.1 * \sum_{j=1}^{n} \operatorname{Sec}_{\mathrm{Num}_{i}}\right)
$$

Where $n$ is the number of medical studies that have been analyzed and the denominator (4) is the maximum DOI. Based on the analysis of the previous studies and previous breast cancer medical reports, like Breast Cancer
Facts \& Figures (2019), Cancer Facts \& Figures (2020), Breast cancer risk factors (2009) and Breast Cancer Risk and Prevention (2019), we supposed that $90 \%$ of the essential risk factor effect and $10 \%$ percent of the secondary risk factors will be summed to constitute the final DOIR value.

The final DOI $\left(\mathrm{DOI}_{\mathrm{i}}^{\mathrm{F}}\right)$ is inferred from the medical questionnaire-based degree of importance $\left(\mathrm{DOI}^{\mathrm{Q}}\right.$ of Table 3 ) and the international medical reports-based degree of importance (DOI ${ }_{\mathrm{i}}^{\mathrm{O}}$ of Table 4) as Equation 5 suggests, while the suggested training weight (STW) in Table 5 is

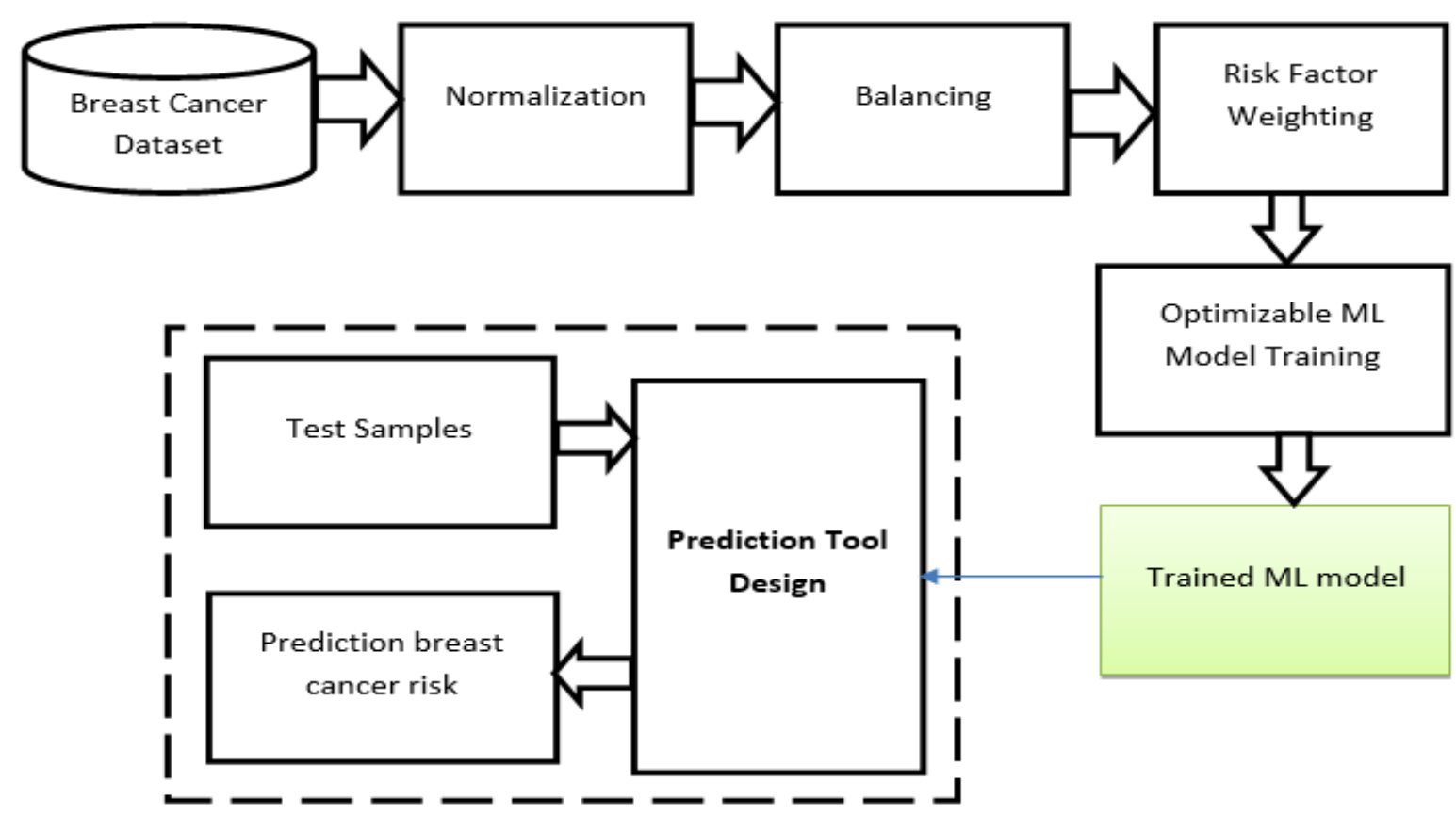

Figure 1. Proposed System Methodology 


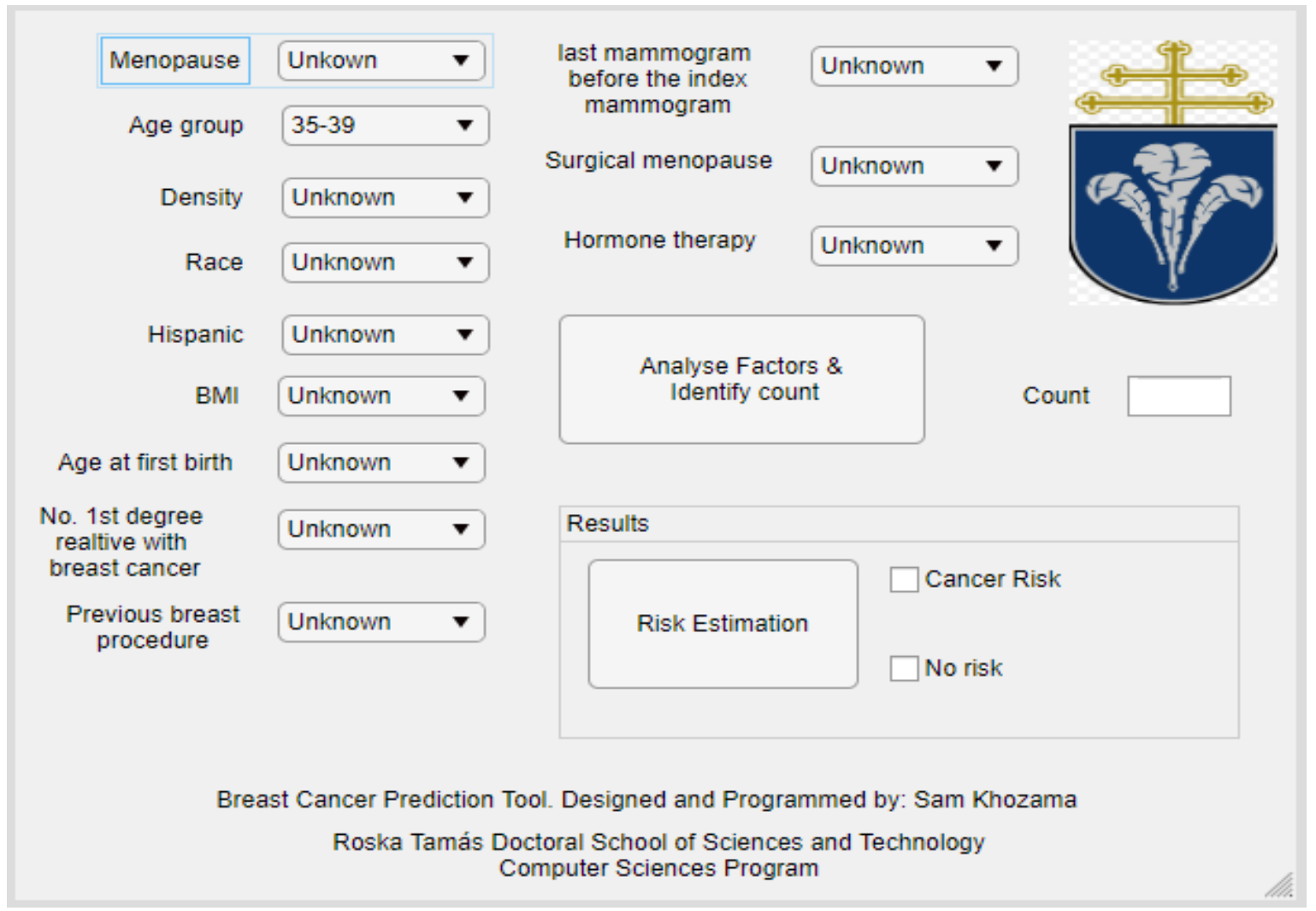

Figure 2. Proposed Breast Cancer Prediction Tool

Table 2. Balancing Approaches and Their Corresponding Classes Percentages

\begin{tabular}{lcccc}
\hline Balancing method & Majority class sample number & Majority class percentage & Minor class sample number & Minor class percentage \\
\hline Oversampling & 271,355 & $85.36 \%$ & 46,525 & $14.64 \%$ \\
Down-sampling & 77,000 & $89.22 \%$ & 9,305 & $10.78 \%$ \\
Mixed & 225,562 & $82.90 \%$ & 46,525 & $17.10 \%$ \\
\hline
\end{tabular}

inferred based on the DOIF (Equation 6).

$$
\begin{aligned}
\operatorname{DOI}_{i}^{F} & =\frac{D O I_{i}^{Q}+D O I_{i}^{R}}{2} \\
S T W_{i} & =\left\{\begin{array}{cl}
\operatorname{round}\left(\operatorname{DOII}_{i}^{F} * 6\right) & \text { if } \operatorname{DOI}_{i}^{F} \geq 0.49 \\
\operatorname{round}\left(\operatorname{DOI}_{i}^{F} * 5\right) & \text { if } \operatorname{DOI}_{i}^{F} \geq 0.39 \\
1 & \text { otherwise }
\end{array}\right\}
\end{aligned}
$$

Table 3. Breast Cancer Risk Factors DOI Based on the Medical Questionnaire

\begin{tabular}{lccccc}
\hline No. & Risk Factor & High & Median & Low & DOIQ \\
\hline 1 & Menopause & $30 \%$ & $47.50 \%$ & $22.50 \%$ & 0.37 \\
2 & Age group & $27.50 \%$ & $62.50 \%$ & $10.00 \%$ & 0.415 \\
3 & Density & $25 \%$ & $45.00 \%$ & $30.00 \%$ & 0.33 \\
4 & Race & $25 \%$ & $40.00 \%$ & $35.00 \%$ & 0.31 \\
5 & Hispanic & $19.40 \%$ & $16.70 \%$ & $63.90 \%$ & 0.183 \\
6 & BMI & $25.60 \%$ & $38.50 \%$ & $35.90 \%$ & 0.307 \\
7 & agefirst & $27.50 \%$ & $45.00 \%$ & $27.50 \%$ & 0.345 \\
8 & nrelbc & $56.40 \%$ & $25.60 \%$ & $17.90 \%$ & 0.44 \\
9 & brstproc & $34.20 \%$ & $23.70 \%$ & $42.10 \%$ & 0.30 \\
10 & lastmamm & $34.20 \%$ & $32.10 \%$ & $33.70 \%$ & 0.33 \\
11 & Surgical & $7.70 \%$ & $30.80 \%$ & $61.50 \%$ & 0.169 \\
& menopause & & & & \\
12 & Hormone & $42.50 \%$ & $37.50 \%$ & $20 \%$ & 0.405 \\
& therapy & & & & \\
\hline
\end{tabular}

The most significant risk factors, as Table 5 describes, are Age group, nrelbc and race while the medium significance risk factors are: Hormone therapy, agefirst, density, Menopause and BM. However, the least essential risk factors are Hispanic, brstproc, lastmamm and surgical menopause.

The effect of weighting the risk factors against the non-weighted version of the dataset is shown in Table 6. The results indicate that the performance increases by $6.9 \%$ after the weighting approach; similarly, the False Discovery Rate (FDR) is minimized by $22.6 \%$ and $3.2 \%$ for the minor and majority class respectively. The False Negative Rate (FNR), as well, is minimized for the majority and minor class by $5 \%$ and $17.6 \%$ respectively. FDR and FNR indicate the percentage of false positives and false negatives respectively (Pawitan et al., 2005).

\section{Discussion}

To check the results shown in Table 6, many test scenarios are suggested by removing one or more essential/non-essential risk factors; so that the optimizable tree-based classifier accuracy, as well as the classification errors, are computed to check the validity of each scenario.

Table 7 illustrates that the weighted version of the dataset has better performance than the non-weighted one. 
Table 4. Breast Cancer Risk Factors DOI Based on the Medical Reports

\begin{tabular}{|c|c|c|c|c|c|c|c|c|c|c|}
\hline \multirow[t]{2}{*}{ No. } & \multirow[t]{2}{*}{ Risk Factor } & \multicolumn{4}{|c|}{ Essential $\uparrow$} & \multicolumn{4}{|c|}{ Secondary } & \multirow[t]{2}{*}{ DOIR } \\
\hline & & 1 & 2 & 3 & 4 & 1 & 2 & 3 & 4 & \\
\hline 1 & Menopause & & & 1 & & 1 & 1 & 1 & & 0.3 \\
\hline 2 & Age group & 1 & 1 & 1 & 1 & & & & & 0.9 \\
\hline 3 & Density & 1 & 1 & & & & & 1 & 1 & 0.5 \\
\hline 4 & Race & 1 & 1 & & 1 & & & & & 0.675 \\
\hline 5 & Hispanic & 1 & & & & & & & 1 & 0.25 \\
\hline 6 & BMI & & 1 & & & 1 & & 1 & 1 & 0.3 \\
\hline 7 & agefirst & & 1 & 1 & & 1 & & & 1 & 0.5 \\
\hline 8 & nrelbc & & 1 & 1 & 1 & 1 & & & & 0.7 \\
\hline 9 & brstproc & & & & & 1 & & 1 & & 0.05 \\
\hline 10 & Lastmamm & & & & & & & & & - \\
\hline 11 & Surgical menopause & & & & & 1 & & & & 0.025 \\
\hline 12 & Hormone therapy & 1 & 1 & & & & & 1 & 1 & 0.5 \\
\hline
\end{tabular}

Table 5. The DOIf of the Breast Cancer Risk Factors

\begin{tabular}{lccc}
\hline No. & Risk Factor & DOIf $\dagger$ & STW \\
\hline 1 & Menopause & 0.3357 & 1 \\
2 & Age group & 0.65751 & 4 \\
3 & Density & 0.4156 & 1 \\
4 & Race & 0.49253 & 3 \\
5 & Hispanic & 0.21659 & 1 \\
6 & BMI & 0.30358 & 1 \\
7 & agefirst & 0.42255 & 2 \\
8 & nrelbc & 0.572 & 3 \\
9 & brstproc & 0.1751 & 1 \\
10 & lastmamm & 0.16511 & 1 \\
11 & Surgical menopause & 0.09712 & 1 \\
12 & Hormone therapy & 0.45254 & 3 \\
\hline
\end{tabular}

$\uparrow$ Numbers 1-12 indicates the weight order.
Weighting the risk factors has increased the performance by $6.9 \%$. The risk factors differ in their degree of importance (i.e., their effect in defining the final risk degree). Table 7 shows that the most effective risk factor is the "Race" factor as the accuracy decreased by $4.3 \%$ after removing this factor. Other risk factors like age at first birth (agefirst), age group, Nrelbc, BMI and Hispanic affect the performance significantly after removing them from the dataset. By removing one of the risk factors (race, age group, agefirst, BMI and Hispanic), an increment in the minor FNR rate is noticed. By removing couples of risk factors like (age and race) or (Nrelbc, age and race) the performance degrades significantly by 6.2 to $7.8 \%$ and the minor class FNR error increases as well by $23 \%$ to $40 \%$, which is a very huge error rate (i.e. these factors are essential). However, some factors like menopause, surgical menopause (surgmeno) and hormone-therapy

\section{Performance of risk estimation model on three different balanced risk database}

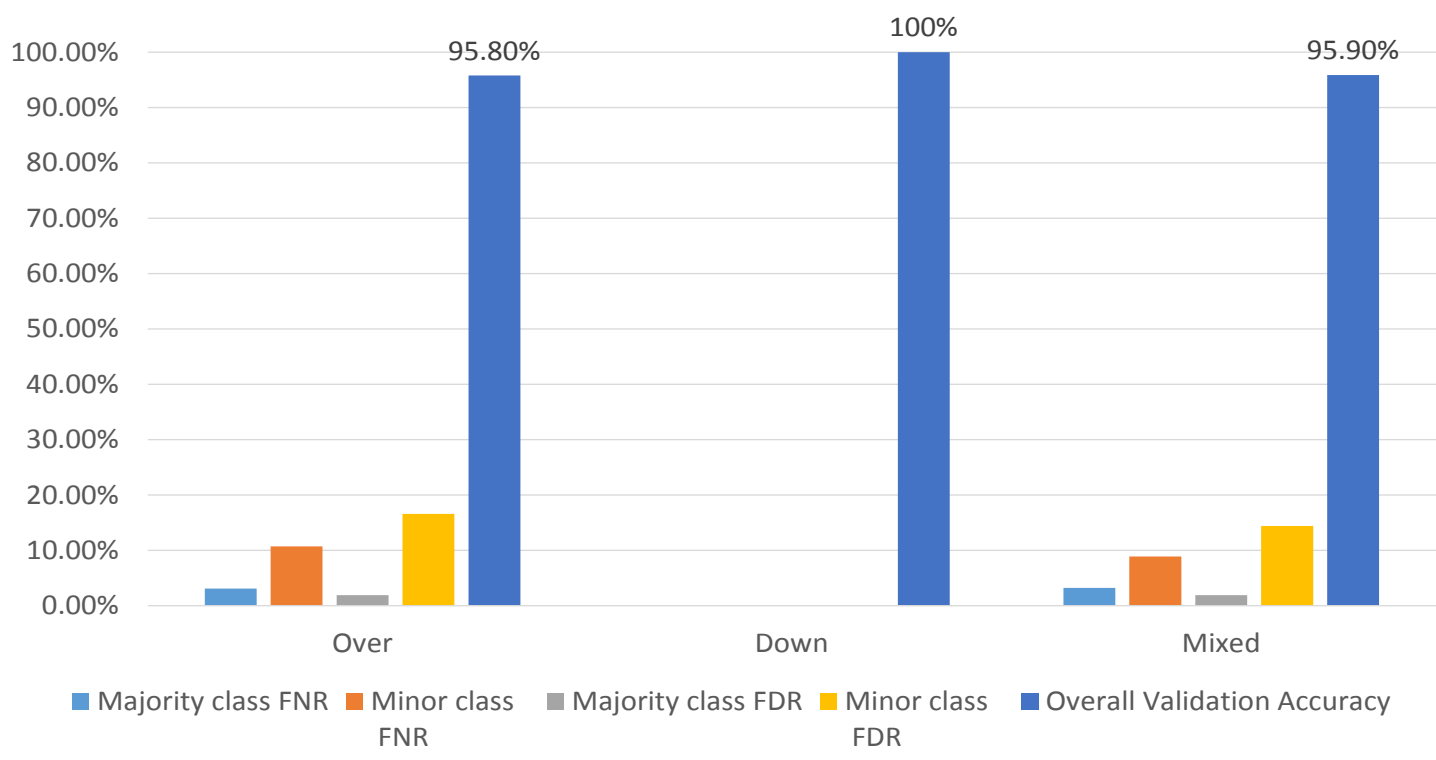

Figure 3. Effect of Scaling Risk Factors on the Performance of Risk Estimation Model on Three Different Balanced Database. 
DOI:10.31557/APJCP.2021.22.11.3543

Breast Cancer Prediction based on Weighted Risk Factors

Table 6. Evaluation of the Risk Estimation Model Using the Weighted and Non-Weighted Version of the Risk Factors.

\begin{tabular}{lcccccc}
\hline Data status & $\begin{array}{c}\text { Majority class } \\
\text { FNR }\end{array}$ & $\begin{array}{c}\text { Minor class } \\
\text { FNR }\end{array}$ & $\begin{array}{c}\text { Majority class } \\
\text { FDR }\end{array}$ & $\begin{array}{c}\text { Minor class } \\
\text { FDR }\end{array}$ & $\begin{array}{c}\text { Overall Validation } \\
\text { Accuracy }\end{array}$ & $\begin{array}{c}\text { Training } \\
\text { Time }\end{array}$ \\
\hline With Weighting & $3.30 \%$ & $10.50 \%$ & $1.80 \%$ & $17.50 \%$ & $95.70 \%$ & 38.65 \\
Without Weighting & $8.30 \%$ & $28.10 \%$ & $5.00 \%$ & $40.10 \%$ & $88.80 \%$ & 41.09 \\
\hline
\end{tabular}

Table 7. Evaluation of the Risk Estimation Model Using Different Selections of the Weighted Risk Factor

\begin{tabular}{lcccccc}
\hline Deleted risk factor & $\begin{array}{c}\text { Majority class } \\
\text { FNR }\end{array}$ & $\begin{array}{c}\text { Minor class } \\
\text { FNR }\end{array}$ & $\begin{array}{c}\text { Majority class } \\
\text { FDR }\end{array}$ & $\begin{array}{c}\text { Minor class } \\
\text { FDR }\end{array}$ & $\begin{array}{c}\text { Overall Validation } \\
\text { Accuracy }\end{array}$ & $\begin{array}{c}\text { Training } \\
\text { Time }\end{array}$ \\
\hline Age & $3.50 \%$ & $16.40 \%$ & $2.80 \%$ & $19.60 \%$ & $94.60 \%$ & 36.05 \\
Race & $6.40 \%$ & $21.60 \%$ & $3.80 \%$ & $32.10 \%$ & $91.40 \%$ & 43.23 \\
Nrelbc & $4.90 \%$ & $16.10 \%$ & $2.80 \%$ & $25.40 \%$ & $93.50 \%$ & 39.09 \\
Hormone Therapy & $4.00 \%$ & $13.10 \%$ & $2.30 \%$ & $21.20 \%$ & $94.70 \%$ & 38.10 \\
surgmeno & $3.90 \%$ & $13.10 \%$ & $2.30 \%$ & $20.80 \%$ & $94.70 \%$ & 38.20 \\
lastmamo & $4.70 \%$ & $15.30 \%$ & $2.70 \%$ & $24.40 \%$ & $93.80 \%$ & 40.32 \\
brstproc & $4.30 \%$ & $13.70 \%$ & $2.40 \%$ & $22.60 \%$ & $94.30 \%$ & 37.12 \\
agefirst & $4.80 \%$ & $17.40 \%$ & $3.00 \%$ & $25.50 \%$ & $93.30 \%$ & 41.37 \\
bmi & $4.90 \%$ & $16.60 \%$ & $2.90 \%$ & $25.70 \%$ & $93.40 \%$ & 39.70 \\
Hispanic & $4.90 \%$ & $16.50 \%$ & $2.90 \%$ & $25.60 \%$ & $93.40 \%$ & 41.37 \\
Density & $4.40 \%$ & $15.10 \%$ & $2.60 \%$ & $23.00 \%$ & $94.10 \%$ & 40.20 \\
menopause & $3.50 \%$ & $11.30 \%$ & $2.00 \%$ & $18.80 \%$ & $95.30 \%$ & 39.20 \\
Age \& Race & $6.60 \%$ & $33.50 \%$ & $5.80 \%$ & $36.60 \%$ & $89.50 \%$ & 36.07 \\
Race \& Nrelbc & $7.80 \%$ & $30.50 \%$ & $5.40 \%$ & $39.50 \%$ & $88.90 \%$ & 36.20 \\
Nrelbc \& Age \& Race & $5.50 \%$ & $50.50 \%$ & $8.40 \%$ & $39.40 \%$ & $87.90 \%$ & 33.59 \\
menopaus \& brstproc \& & $5.40 \%$ & $20.90 \%$ & $3.70 \%$ & $28.50 \%$ & $92.30 \%$ & 33.70 \\
surgmeno & & & & & \\
\hline
\end{tabular}

decrease the accuracy by a small range $(0.4 \%$ to $1 \%)$. From another point of view, missing the three risk factors (menopause, brstproc and surgmeno) decreases the accuracy only by $3.4 \%$. So these factors have less impact than others on defining the last risk degree, and in order to validate this conclusion, a down-weight approach was applied in which each weak-impact risk factor is weighted by a less-than- 1 factor $(0.2,0.3,0.5$, etc. $)$ and the results are listed in Table 8. Scaling menopause, for example, by 0.5 improves the validation accuracy by $0.1 \%$. Scaling the other low-important risk factors also improves the accuracy by $0.1 \%$ and reduces the FNR error by $0.2 \%$. However, in some cases; it increases the FNR of the minor class (and this is because the minor class percentage is small), but at the same time the FDR rate has been decreased by $(0.5-0.9 \%)$.

The same scaling technique used on the oversampled dataset; is applied to the down-sampled and the mixed ones, Figure 3 includes a detailed comparison of the performance of scaling choice $($ age $=4$, race $=3$, agefirst $=2$, nrelbc $=3$, current hormone therapy (current hor $)=3$, menopause $=0.5$, density $=0.3$, brstproc $=0.2$, lastmamm $=0.3$, surgmeno $=0.2$ ) over the three balanced datasets. Figure 3 shows that the down-sampled dataset has the highest accuracy $(100 \%)$ and the least error rates $(0 \%)$; however, this down-sampled dataset has a volume of $27.15 \%$ only compared with the over-sampled version. So although the down-sampled dataset has the best accuracy, the over-sampled and the mixed versions have better performance since they consist of a much larger number

Table 8. The Effect of Down-Weighting the Weak-Impact Risk Factors on the Performance of Risk Estimation Model on the Oversampled Risk Database

\begin{tabular}{lccccc}
\hline Down-scaling & $\begin{array}{c}\text { Majority } \\
\text { class FNR }\end{array}$ & $\begin{array}{c}\text { Minor } \\
\text { class } \\
\text { FNR }\end{array}$ & $\begin{array}{c}\text { Majority } \\
\text { class FDR }\end{array}$ & $\begin{array}{c}\text { Minor } \\
\text { class } \\
\text { FDR }\end{array}$ & $\begin{array}{c}\text { Overall } \\
\text { Validation } \\
\text { Accuracy }\end{array}$ \\
\hline menopause $=0.5$ & $3.10 \%$ & $10.80 \%$ & $1.90 \%$ & $16.60 \%$ & $95.80 \%$ \\
menopause $=0.5$, brstproc $=0.2$ & $3.10 \%$ & $10.70 \%$ & $1.90 \%$ & $17.00 \%$ & $95.80 \%$ \\
menopause $=0.5$, brstproc $=0.2$, , lastmamm $=0.2$ & $3.20 \%$ & $11.50 \%$ & $2 \%$ & $17.50 \%$ & $95.60 \%$ \\
brstproc $=0.2$, lastmamm $=0.3$, surgmeno $=0.2$ & $3.30 \%$ & $10.10 \%$ & $1.80 \%$ & $17.60 \%$ & $95.70 \%$ \\
menopause $=0.5$, brstproc $=0.2$, lastmamm $=0.3$,surgmeno $=0.2$ & $3.10 \%$ & $11 \%$ & $1.90 \%$ & $17 \%$ & $95.70 \%$ \\
menopause $=0.5$,Density $=0.3$, brstproc $=0.2$, lastmamm $=0.3$, & $3.10 \%$ & $10.70 \%$ & $1.90 \%$ & $16.60 \%$ & $95.80 \%$ \\
surgmeno $=0.2$ & & & & & \\
\hline
\end{tabular}


of samples so that the new test samples will be classified more correctly.

In this research, the effect of weighting and selection of the risk factors has been studied. In addition, three versions of the balanced dataset were tested. The experiments proved that the weighting technique improved the accuracy and reduced the errors significantly. In future work, the weighting model will be used to generate a fuzzy risk factor score in the range (0-100) instead of a scalar risk score.

\section{Author Contribution Statement}

Khozama S. proposed the idea and design of the work. Mayya A. collaborated in editing the layout of the paper and coding some parts of the software. Both authors contributed in formulating the mathematical equations, writing the manuscript, and testing the software.

\section{Acknowledgements}

\section{Funding Statement}

This work was supported by Pázmány Péter Catholic University, Budapest, Hungary. The research project has been partially supported by the European Union, co-financed by the European Social Fund through the grant EFOP-3.6.3-VEKOP-16-2017-00002.

\section{Approval}

The current study deals with secondary public data (breast cancer risk factors), so it doesn't need from approval of scientific body. This paper is a part of the dissertation submitted in fulfillment of the requirements for the degree of Doctor of Philosophy in Pázmány Péter Catholic University, Budapest, Hungary.

\section{Ethics approval}

Dataset for this study is completely public; therefore, no ethical approval is required.

\section{Availability of data}

Data collection and sharing was supported by the National Cancer Institute-funded Breast Cancer Surveillance Consortium (HHSN261201100031C), available at: http://www.bcsc-research.org/.

The study is not registered in any registering dataset.

\section{Conflict of interest}

The authors declared no conflicts of interest.

\section{References}

Ahmad AS, Mayya AM (2020). A new tool to predict lung cancer based on risk factors. Heliyon, 6, e03402.

American Cancer Society (2019). Breast Cancer Facts \& Figures 2019-2020. Atlanta: American Cancer Society.

American Cancer Society. Breast Cancer Risk and Prevention (2019). Atlanta: American Cancer Society.

American Cancer Society. Breast Cancer Fact Sheet (2020). Atlanta: American Cancer Society.

Apté C, Weiss S (1997). Data mining with decision trees and decision rules. Future Gener Comput Syst, 13, 197-210.
Barlow, WE, White E, Ballard-Barbash R, et al (2006). Prospective breast cancer risk prediction model for women undergoing screening mammography. J Natl Cancer Inst, 98, 1204-14.

Bernal JL, Cummins S, Gasparrini A (2017). Interrupted time series regression for the evaluation of public health interventions: A tutorial. Int J Epidemiol, 46, 348-5.

Breast Cancer Surveillance Consortium (BCSC) (2018). Available at: https://breastscreening.cancer.gov/. (Accessed: 5th January 2021).

Colditz GA, Wei EK (2015). Risk prediction models: applications in cancer prevention. Curr Epidemiol Rep, 2, 245-0.

Faith MF (2020). A comparative analysis of breast cancer detection and diagnosis using data visualization and machine learning applications. Healthcare, 8, 1-23.

Hou C, Zhong X, He P, et al (2020). Predicting breast cancer in Chinese women using machine learning techniques: algorithm development. JMIR Med, 8, e17364.

Kamal V, Kumari D (2020). Use of artificial intelligence/machine learning in cancer research during the COVID-19 pandemic. Asian Pac J Cancer Care, 5, 251-3.

Kelleher JD, Namee B, D'arcy A (2020). Fundamentals of machine learning for predictive data analytics: algorithms, worked examples, and case studies. MIT press.

Kourou K, Exarchos TP, Exarchos KP, Karamouzis MV, Fotiadis DI (2015). Machine learning applications in cancer prognosis and prediction. Comput Struct Biotechnol J, 13, 8-17.

LAKY Z (2020). Cancer prevention: Modifiable risk factors. Policy Department for Economic, Scientific and Quality of Life Policies IPOL. doi:10.2861/169496.

Mantovani RG, Horváth T, Cerri R, et al (2018). An empirical study on hyperparameter tuning of decision trees. arXiv e-prints, arXiv-1812.

Ming C, Viassolo V, Probst-Hensch N, et al (2020). Machine learning-based lifetime breast cancer risk reclassification compared with the BOADICEA model: impact on screening recommendations. Br J Cancer, 123, 860-7.

Mochen L, Sundararajan R (2018). Application of Machine Learning Algorithms on Breast Cancer Dataset. Proc. 2018 Electrostatics Joint Conference.

National Breast and Ovarian Cancer Centre. Breast cancer risk factors: a review of the evidence. National Breast and Ovarian Cancer Centre, Surry Hills, NSW, 2009.

Oyewola D, Hakimi D, Adeboye K, Shehu M (2017) Using five machine learning for breast cancer biopsy predictions based on mammographic diagnosis. Int J Eng Technol, 2, 142-5.

Patil S, Moafa IH, Mosa Alfaifi M, et al (2020). Reviewing the Role of Artificial Intelligence in Cancer. Asian Pac J Cancer Biol, 5, 189-99.

Pawitan Y, Michiels S, Koscielny S, Gusnanto A, Ploner A (2005). False discovery rate, sensitivity and sample size for microarray studies. Bioinformatics, 21, 3017-24.

Peng Z, Wei J, Lu X, et al (2016). Treatment and survival patterns of Chinese patients diagnosed with breast cancer between 2005 and 2009 in Southwest China: an observational, population-based cohort study. Medicine, 95, 1-11.

Rajendran K, Jayabalan M, Thiruchelvam V (2020). Predicting breast cancer via supervised machine learning methods on class imbalanced data. Int J Adv Comput Sci Appl, 11, 54-63.

Shieh Y, Hu D, Ma L, et al (2016). Breast cancer risk prediction using a clinical risk model and polygenic risk score. Breast Cancer Res Treat, 159, 513-25.

Somasundaram A, Reddy US (2016). Data imbalance: Effects and solutions for classification of large and highly imbalanced data. In Proceedings of ICRECT, 16, pp 28-34.

Teja KS, Manjunath G, Dekker A, Wee L (2020). Robust Estimation of Breast Cancer Incidence Risk in Presence of 
Incomplete or Inaccurate Information. Asian Pac J Cancer Prev, 21, 2307-13.

Vard A, Firouz AF, Sehhati M, Mohebian M (2018). An optimized framework for cancer prediction using immune signature. J Med Signals Sens, 8, 161-9.

Wang H, Yoon WS (2015). Breast cancer prediction using data mining method. In Proceedings of the 2015 Industrial and Systems Engineering Research Conference, Nashville USA.

Westerdijk L (2018). Predicting Malignant Tumor Cells in Breasts; Master Business Analytics; Vrije University Amsterdam, The Netherlands.

Williams GS, Cubiella J, Griffin SJ (2016) Risk prediction models for colorectal cancer in people with symptoms: A systematic review. BMC Gastroenterol, 16, 63.

Zhong X, Luo T, Deng L, et al (2020). Multidimensional machine learning personalized prognostic model in an early invasive breast cancer population-based cohort in China: Algorithm Validation Study. JMIR Med, 8, e19069.

This work is licensed under a Creative Commons AttributionNon Commercial 4.0 International License. 